\author{
${ }^{1}$ D. Sokolov ${ }^{*},{ }^{1}$ A. Drobyshev, ${ }^{2}$ M. Ramos \\ ${ }^{1}$ IETP, Departament of physics and technology, \\ al-Farabi Kazakh National University, al-Farabi 71, \\ Almaty 050012, Kazakhstan; \\ ${ }^{2}$ Universidad Autonoma de Madrid (U.A.M.),Facultad de Ciencias, \\ Departamento de Física de la MateriaCondensada, Cantoblanco, E-28049, \\ Madrid, Spain. \\ *e-mail: Dmitriy.Sokolov@kaznu.kz
}

\title{
The phase transition between the orientational glass and plastic crystal in cryovacuum condensate of ethanol
}

\begin{abstract}
After more than 100 years of research, the nature of glasses (and disordered condensed matter, in general) and glass transition-related phenomena is still an open issue in condensed-matter physics [1] and the chemicophysical sciences [2]. Thin films of cry vacuum condensates of ethanol formed by condensation of the gas on a cooled metal substrate are studied by infrared spectrometry and thermal desorption techniques. The main purpose was to examine dynamic relaxation processes near the glass transition temperature and the transition from orientation ally disordered to orders states in thin ethanol cry condensate films. Based on the analysis of the thermo grams conclusions that the phase transition orientation glass-plastic crystal of ethanol around $\mathrm{T}=97 \mathrm{~K}$ is reversible and is a phase transition type I.

Key words: SCL - supercooled liquid, crystal plastic, monoclinic crystal, glass transition temperature, IR spectrum, ethanol, cryocrystall.
\end{abstract}

\section{Introduction}

The dynamics of glass-forming liquids thoroughly studied in recent years, both from a theoretical point of view, and from the experimental positions. The dominant dynamic processes governing the behavior of glass-forming materials, is the so-called alpha-relaxation [3], which is characterized by structural relaxation. From the experimental point of view, the alpharelaxation is manifested in the universal features: deviation from quasi-Debye behavior and the rapid increase in the relaxation time with decreasing temperature. The relaxation time is not Arrhenius temperature dependence and can be parametrically described by an empirical law Fogera-TammanFulcher.

Low-temperature forms of super cooled liquids and the corresponding glassy states are of extraordinary interest in this area. Systems with hydrogen bonds, in particular water and ethanol, occupy a special place among the intensely studied glassy materials. Despite extensive research and a (C) 2014 al-Farabi Kazakh National University large number of publications, there are still some unclear, controversial questions relating to the dynamics of vitrification and glass transitions. We note that most of the work over the last 20-30 years deals with solid ethanol produced by quenching (ultrafast cooling from the liquid phase). We have pointed this fact out before and used it as a starting point for comprehensive studies of the properties of thin films of solid ethanol produced during cry vacuum condensation from the gaseous phase on substrates with different cry deposition temperatures. These infrared spectrometry studies, in particular observations of thermally stimulated changes in the signal from an interferometer at a fixed characteristic vibrational frequency of ethanol, show that the structural and thermodynamic measurements are qualitatively consistent $[4,5]$.

Figure 1shows that the typical changes in the interferometer signal at $v=3105 \mathrm{~cm}^{-1}$ with heating $[6,7]$ (lower curve) are in good agreement with the temperature intervals of the structural transformations in a monolithic sample (the diagram in the upper part of the figure). The sample consists of a

Printed in Kazakhstan 
film of thickness $\mathrm{d}=4 \mathrm{mcm}$ condensed from the gaseous phase of ethanol with a substrate temperature of $\mathrm{T}=16 \mathrm{~K}$. The condensation pressure is $\mathrm{P}=4 * 10^{-5}$ Torr. The observations during the heating process were made at a frequency of $v=$
$3105 \mathrm{~cm}^{-1}$. The observed correlation between the behavior of the interferometer signal and the phase diagram of ethanol justified interpreting the IR spectrometry data by matching them to the diagram in the figure.

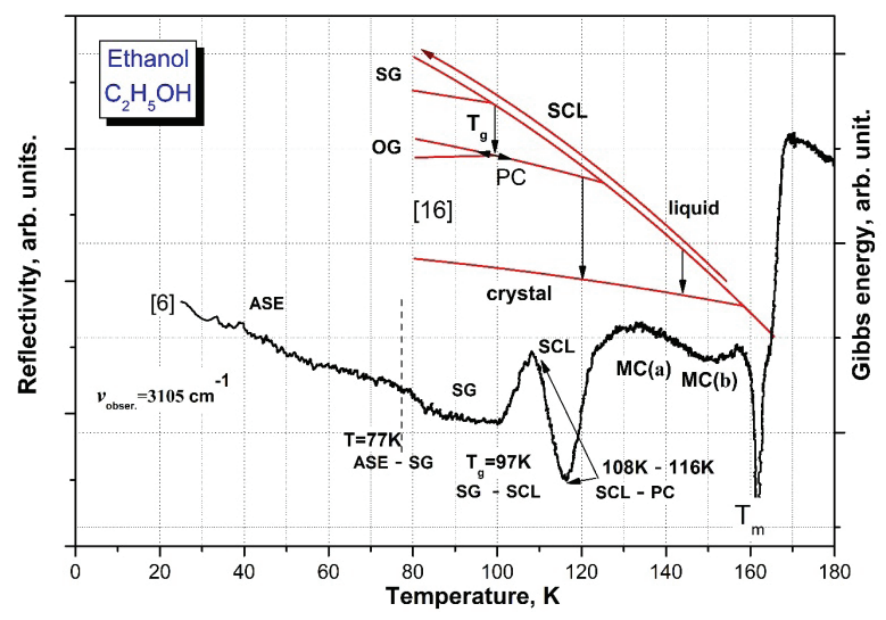

Figure 1 - Comparison of the heating curve for a thin film of ethanol cry condensate (lower curve) with the phase diagram for a monolithic sample obtained from the liquid phase (the diagram in the upper part of the figure).

The phase diagram of ethanol shown in Fig. 1clearly reveals one of its unusual features: near $\mathrm{T}$ $=97 \mathrm{~K}$ there are several states of solid ethanol and transitions of different kinds between them. The following notation is used in the figure: $\mathrm{SG}$ (structured) glass; SCL super cooled liquid; OG orientational glass; ASE amorphous solid ethanol; PC plastic crystal; MC(a) monoclinic crystal (aphase); $\mathrm{MC}(\mathrm{b})$ monoclinic crystal (b-phase); Tg temperature of the glass transition (vitrification); and, Tm melting temperature. Based on these results the following are experimental data, which confirm the following fact.

On cooling, the plastic crystal PC at 105-110K undergoes a transformation into orientational glass (OG) at $\mathrm{T}=97 \mathrm{~K}$, which differs from the $\mathrm{PC}$ in having frozen rotational degrees of freedom. This transition is reversible.

\section{Experiment and data}

Here we present the results of IR spectrometric and thermal desorption studies of thin films of cry vacuum condensates of ethanol produced by condensing the gas at different temperatures and pressures on a cooled metal substrate. The condensation temperature was $\mathrm{T}=16 \mathrm{~K}$. The pressure of the gaseous phase during cry deposition was varied from $\mathrm{P}=4.5^{*} 10^{-5}$ to $8^{*} 10^{-6}$ Torr. The film thickness was set within the range of $1-4$ mcm. The spectral measurements were made over a range of $400-4200 \mathrm{~cm}^{-1}$. The experimental apparatus and techniques have been described previously[6, 7].Here we mention only the basic methods of obtaining the data: laser-interferometric measurements of the rate of condensation, thickness, and refractive index of the thin films of ethanol cry vacuum condensate; IR spectrometric analysis of the transformation processes in the ethanol samples; and, determination of the parameters of the transitions in solid ethanol by the thermal desorption method. 99.99\% pure dry ethanol was used in the experiments.

The experiments were conducted in the following order: the vacuum chamber (a 420-mmdiam cylinder with a height of $400 \mathrm{~mm}$ ) was pumped down by a Turbo-V 301 turbo molecular pump to a pressure of about $10^{-8}$ Torr. A mirror substrate (silver coated copper) with a diameter of $40 \mathrm{~mm}$ attached to the second step of a GiffordMcMahon micro cryogenic system was cooled to $\mathrm{T}$ $=16 \mathrm{~K}$. Gaseous ethanol was fed into the vessel to the condensation pressure $\mathrm{P}=5^{*} 10^{-5}$ Torr through a leak valve. Once the required film thickness was 
obtained the gas feed was shut off and the IR reflection spectrum of the sample was measured. Then the spectrometer was set to a fixed frequency corresponding to characteristic vibrations of the ethanol molecule and the sample was slowly heated while the spectrometer signal at the observation frequency was measured. The samples were heated at a rate of $0.05 \mathrm{~K} / \mathrm{s}$.

Figure 2 shows the behavior of the sample thermogram solid ethanol at a maximum tempera- ture warming $\mathrm{T}=105 \mathrm{~K}$. This temperature corresponds to the formation of the plastic crystal (PC) of the SCL, which is accompanied by a sharp fall in the interferometer signal. As we have previously shown in [7], this corresponds to a considerable broadening of the absorption band and reducing reflectivity of the surface substrate film when the film is a plastic ethanol crystal. These facts reflect the change in the signal at that frequency spectrometer observation.

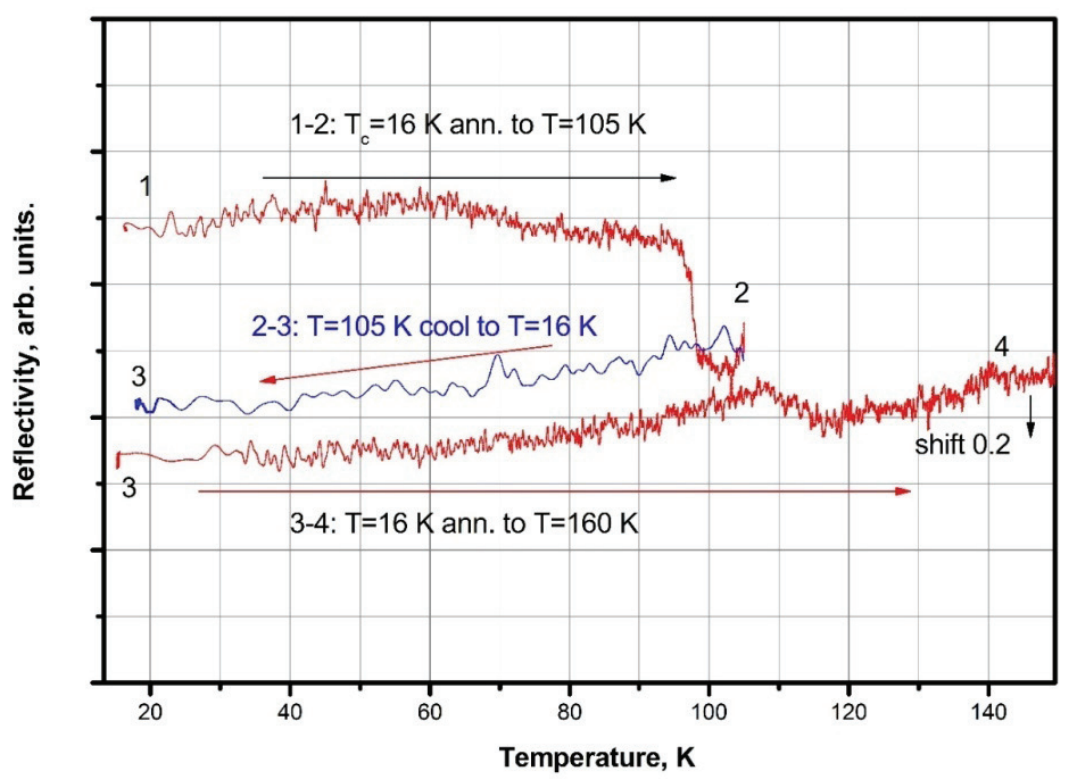

Figure 2 - Temperature dependence of the IR spectrometer signal.

The condensation temperature is $16 \mathrm{~K}$, the condensation pressure is $5^{*} 10^{-5} \mathrm{Torr}$, the film thickness is $3.7 \mathrm{mcm}$, and the observation frequency is $3105 \mathrm{~cm}^{-1}$. The final heating and stabilization temperature for the substrate is $105 \mathrm{~K}$.

The start of the experiment corresponds to position 1, where an ethanol cry condensate film with a thickness $d=4 \mathrm{mcm}$ was condensed from the gaseous phase onto a substrate at $\mathrm{T}=16 \mathrm{~K}$. After condensation the IR spectrum was measured and the IR spectrometer was set to an observation frequency of $v=3105 \mathrm{~cm}^{-1}$ corresponding to the amplitude at half-width for absorption by the $\mathrm{O}-\mathrm{H}$ bond. Observation interval 1-2. Heating the sample to $\mathrm{Tj} 105 \mathrm{~K}$ at a rate of $0.05 \mathrm{~K} / \mathrm{s}$. Heating is stopped on reaching $\mathrm{T}=105 \mathrm{~K}$, which corresponds to the temperature at which the PC exists. The slight increase in the interferometer signal corresponded to the onset of the transition of the plastic crystal into the monoclinic crystal. This process is slowed down when the sample is cooled.

General analysis thermo gram 1-2 leads to the following conclusions. In the temperature range 80-90
$\mathrm{K}$ amorphous solid ethanol (ASE) goes into the structural glass (SG). In the temperature range 95$98 \mathrm{~K}$, the transition structural glass - super cooled liquid (SG-SCL). At T=98K, the abrupt change in the spectrometer signal, associated with the transition super viscous liquid-crystal plastic (SCL-PC).

Observation interval 2-3. Cooling a sample in the $\mathrm{PC}$ state to $\mathrm{T}=16 \mathrm{~K}$ at a rate of $0.1 \mathrm{~K} / \mathrm{s}$. According to the phase diagram for a monolithic sample, at $97 \mathrm{~K}$ there should be a PC-OG transition. This process is accompanied by "freezing" of the rotational degrees of freedom of the ethanol molecule in a cubic lattice, which should not affect the observed frequencies. This can be seen in Figure 3.

Observation interval 3-4. Heating the orientational glass $\mathrm{OG}$ from $16 \mathrm{~K}$ to the melting point at $160 \mathrm{~K}$. For convenience the thermo gram 
for 3-4 is shifted relative to that for $2-3$ in the overlay of the thermo grams for $2-3$ and $3-4$ by 0.2 nominal units (shift indicated by an arrow).

The thermo gram with cooling and warming in the temperature range $16-105 \mathrm{~K}$ coincide. Thus, we can conclude that we have experimentally observed reversibility of the PC OG PC. Sudden change of the spectrometer signal at $\mathrm{T}=110 \mathrm{~K}$ corresponds to the transition of the plastic crystal (PC) in the state of monoclinic crystal.

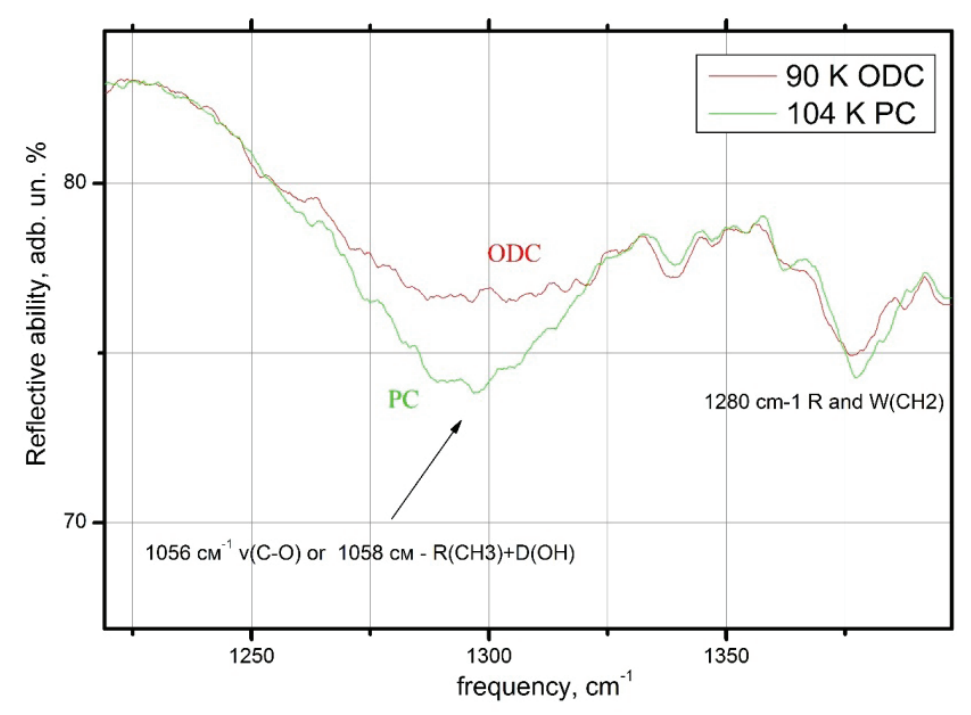

Figure 3 - IR spectra of the ethanol cryogenic condensate 4 mcm thick. After condensation at $T=16 \mathrm{~K}$, after heating from $T=16 \mathrm{~K}$ to $104 \mathrm{~K}$, and (3) after cooling from $T=104 \mathrm{~K}$ to $90 \mathrm{~K}$.

\section{References}

1. Anderson P.W. Glassy state problem // Science. - 1995. - Vol.267. - 1615 p.

2. Zallen R. The Physics of Amorphous Solids. -New York: Wiley, 1983. - P. 49-72.

3. Talon C., Ramos M., and Vieira S. Low-temperature specific heat of amorphous, orientational glass, and crystal phases of ethanol // Physical Review B. -2002. - Vol.66. - P.012201-2.

4. Ramos M., Viera S. Quantitative Assessment of the Effects of Orientational and Positional Disorder on Glassy Dynamics//Phys. Rev. - 1997. - Vol.78. - N. 1. - 82. p
5. Haida O., Suga H., and Seki S. Calorimetric study of the glassy state XII. Plural glass-transition phenomena of ethanol // w-521, Journal Chemistry Thermodynamics. - 1977. - Vol.9. - P.1133-1148.

6. Drobyshev A., Aldiyarov A., Zhumagaliuly D., Kurnosov V., Tokmoldin N. Thermally stimulated transformations in cry vacuum water ices //Low temperature physics. - 2007. -Vol.33. N. 4. -P.479-487.

7. Drobyshev A., Abdykalykov K., Aldiyarov A., Kurnosov V., Tokmoldin N., and Zhumagaliuly D. IR spectra of water polyaggregates in a nitrogen cry matrix // Low temperature physics. - 2007. Vol.33. -N. 8. - P.916-922. 